\title{
L'hydraulique en plein air. Le cas des expériences sur les dépenses d'eau
}

A case of "out-of-doors" experiments: the hydraulic experiments on the discharge of water by Poncelet et Lesbros

Bruno Belhoste

\section{(2) OpenEdition}

12 Journals

\section{Édition électronique}

URL : http://journals.openedition.org/dht/1586

DOI : 10.4000/dht.1586

ISSN : 1775-4194

Éditeur :

Centre d'histoire des techniques et de l'environnement du Cnam (CDHTE-Cnam), Société des élèves du CDHTE-Cnam

Édition imprimée

Date de publication : 1 décembre 2011

Pagination : 15-27

ISBN : 978-2-9530779-7-1

ISSN : 0417-8726

\section{Référence électronique}

Bruno Belhoste, «L'hydraulique en plein air. Le cas des expériences sur les dépenses d'eau »,

Documents pour I'histoire des techniques [En ligne], 20 | 2e semestre 2011, mis en ligne le 13 juin 2012, consulté le 08 septembre 2020. URL : http://journals.openedition.org/dht/1586 ; DOI : https://doi.org/ 10.4000/dht.1586 


\title{
L'hydraulique en plein air Le cas des expériences sur les dépenses d'eau
}

\section{Bruno Belhoste}

\author{
Université Paris 1 - Panthéon-Sorbonne - EA 127 Modernités et Révolutions
}

\begin{abstract}
Résumé
Les expériences sur les dépenses d'eau menées à Metz par Poncelet et Lesbros en 1827-1829 grâce à un financement du gouvernement, fournissent un exemple particulièrement remarquable de recherche expérimentale en plein air. Elles se distinguent par la taille du dispositif, la rigueur du protocole, la précision des mesures et l'ampleur du compte-rendu, qui donne lieu à un mémoire rédigé par Poncelet et publié dans le recueil des Savants étrangers de l'Académie. Bien qu'elles n'aient pas marqué une grande avancée scientifique, elles ont exercé une grande influence sur le développement ultérieure de l'hydraulique et de la mécanique appliquée en servant de modèles pour les grandes expériences en vraie grandeur en France et ailleurs.
\end{abstract}

Résumés et mots-clés en anglais sont regroupés en fin de volume, accompagnés des mots-clés français

$E^{n}$ 1827, les officiers du génie Poncelet et Lesbros entreprennent à Metz une série d'expériences en grand concernant les lois de l'écoulement des eaux par des orifices. Nous chercherons ici à reconstituer précisément leur démarche, l'organisation des expériences et les résultats obtenus à partir du compte rendu exceptionnellement précis qu'ils en ont donnél. Sans être négligeables, ces expériences n'ont pas représenté une grande avancée scientifique. Elles sont tombées aujourd'hui quasiment

1 J.-V. Poncelet et J. Lesbros, « Expériences hydrauliques sur les lois de l'écoulement de l'eau a travers les orifices rectangulaires verticaux a grandes dimensions, Mémoire lu à l'Académie des sciences le lundi 16 novembre 1829 ", Mémoires présentés par divers savans à l'Académie royale des sciences de l'Institut de France, t. 3, 1832, pp. 242-501 (cité plus loin comme «Expériences hydrauliques »). Le mémoire a été également vendu en tiré-à-part, avec une autre pagination, chez Bachelier. dans l'oubli. Leur intérêt réside ailleurs. Le travail des deux officiers fournit en effet une très bonne illustration d'une tradition expérimentale féconde et originale, celle des ingénieurs hydrauliciens, dont les méthodes et les rendus étaient très différents de celles de physiciens expérimentateurs, beaucoup plus étudiées par les historiens des sciences.

L'expérimentation est une activité multiforme, qui peut se déployer dans toute sorte d'espaces géographiques et sociaux. Si le cas paradigmatique a été longtemps le laboratoire, les études sur les sciences, en particulier les études historiques, ont exhibé bien d'autres cadres propices à l'activité expérimentale, certains spécialisés, comme l'observatoire, le cabinet d'histoire naturelle ou l'amphithéâtre universitaire, d'autres profanes, comme la manufacture et l'hôpital. Tous ces lieux se distinguent par leur caractère clos et permanent. ॥ existe cependant des espaces plus ouverts et plus fugitifs pour l'expérimentation. II peut s'agir d'une 


\section{L'hydraulique en plein air}

salle quelconque, dédiée temporairement à une expérience. II est possible aussi que le dispositif soit, tout simplement, en plein air. Comme le lecteur aura l'occasion de le constater en parcourant ce numéro, et en consultant la littérature sur le sujet, le terrain de l'expérimentation peut être, en effet, un chantier, une route, un champ de bataille, la surface de la mer, le sommet d'une montagne ou l'espace interplanétaire? ${ }^{2}$.

Tout lieu, au fond, dispose d'un potentielen matière d'expérimentation. Encore faut-il qu'il soit équipé pour cela. L'expérimentation exige en effet un dispositif, qui se décline matériellement et socialement, avec des arrangements spatiaux, des participants, actifs ou passifs, et des instruments. C'est ce dispositif, et lui seul, qui transforme un lieu quelconque en espace d'expérimentation et permet le travail d'épuration, d'abstraction et d'universalisation par lequel l'évènement devient preuve expérimentale. Outre les opérations matérielles et intellectuelles sur le lieu de l'expérimentation, ce travail comprend des investigations préliminaires, qui contribuent au choix du site, et des prolongements, qui visent à stabiliser la preuve et à publiciser le résultat.

Ce schéma général se décline de multiples manières en fonction des objets, des acteurs, des ressources et des espaces. Or l'historiographie a privilégié, aux dépens de toutes les autres, l'expérimentation pratiquée dans la tradition de la philosophie naturelle, d'où l'accent mis sur le laboratoire, alors qu'en réalité, loin de se réduire à un développement linéaire et uniforme, I'histoire des pratiques expérimentales est foisonnante et multiforme. Le préalable à une meilleure appréciation de ce qu'a été l'expérimentation dans le passé est donc d'analyser sur des exemples précis d'autres modalités de l'activité expérimentale, avec d'autres types d'acteurs et d'autres types de lieu, en somme de mettre en évidence, par des études de cas, l'importance et la vitalité d'autres cultures expérimentales.

De ce point de vue, les expériences d'hydraulique de Poncelet et Lebros sont riches d'enseignements. Elles montrent que l'expérimentation en plein air et en grand a pu présenter des caractères que l'on associe habituellement à l'expérimentation physique en espace confiné, comme le contrôle et la précision. Pour autant, comme on verra, les différences entre les deux styles expérimentaux apparaissent aussi de façon éclatante, tant pour le cadre et de la conduite des

2 Sur les sciences de terrain, voir H. Kuklick and R. E. Kohler éd., Science in the Field, Osisis, vol. 11, 1996. expériences que pour les objectifs poursuivis et les modes de validation et d'exposition des résultats. Le travail des deux hydrauliciens illustre en effet une certaine culture expérimentale, très spécifique aux ingénieurs et particulièrement vivace en France entre 1750 et $1850^{3}$.

\section{L'expérience en grand}

Au début de 1827 le général Sabatier, commandant de l'École de Metz, demande au professeur de mécanique appliquée aux machines Jean-Victor Poncelet (1788-1867), déjà connu pour ses travaux en géométrie et en mécanique, de reprendre et de continuer " pour l'instruction des élèves et pour l'avancement des sciences "les expériences hydrauliques entreprises à Mézières au XVIII ${ }^{e}$ siècle ${ }^{4}$. Poncelet donne aussitôt son accord et s'adjoint l'officier du génie Joseph-Aimé Lesbros (1790-1860), un ancien camarade d'école qui l'a déjà aidé au cours des années précédentes dans ses expériences sur les roves à aubes. Dans l'esprit du général Sabatier, il s'agit surtout d'une opération de prestige pour les armes savantes et leur école. Dans celui de Poncelet, le but est plutôt de compléter des recherches sur les dépenses d'eau commencées les années précédentes à l'occasion de ses travaux sur les roves hydrauliques. Grâce à l'appui du président du Comité des fortifications, Sabatier obtient en août 1827 l'autorisation du Ministère de la guerre et des fonds pour financer les recherches.

Voici comment Poncelet lui-même en a résumé l'objet :

« Étudier, principalement pour les besoins de la pratique et à l'aide de l'expérience, les lois de l'écoulement de l'eau à travers les orifices rectangulaires verticaux, limités vers la partie supérieure par une vanne mobile

(1) dans l'hypothèse des minces parois, où l'eau s'échappant librement dans l'air, l'orifice se trouve complètement isolé des faces latérales et du fond du réservoir :

(2) dans l'hypothèse où l'orifice avoisine plus ou moins ces faces et ce fond, d'ailleurs disposés

3 Je remercie Olivier Darrigol pour sa lecture critique qui m'a permis de corriger ou d'améliorer certains points de cet article. 4 Sur l'École de l'artillerie et du génie de Metz, voir B. Belhoste et A. Picon dir., L'École d'application de l'artillerie et du génie de Metz (1802-1870). Enseignement et recherches, Paris, Musée des Plans-Reliefs, 1996. Sur l'enseignement de Poncelet, voir K. Chatzis, « Fabriquer et recevoir un cours magistral. Les cours de mécanique appliquée de Jean-Victor Poncelet à l'École de l'Artillerie et du Génie et à la Sorbonne ॥, 18251848, Histoire de l'éducation, vol. 120, 2008, pp. 113-138 
perpendiculairement ou obliquement par rapport à la paroi qui contient l'orifice :

(3) dans l'hypothèse des parois épaisses, où l'eau serait immédiatement reçue dans un coursier ou canal d'une petite longueur, découvert à la partie supérieure, et qui formerait, ou non, le prolongement exact des bords de l'orifice, en variant du reste les expériences suivant les autres dispositions indiquées dans les deux premiers articles:

(4) étudier, mais subsidiairement, les lois physiques ou mathématiques de chaque phénomène, et les causes qui produisent des écarts entre l'expérience et les formules en usage pour calculer la vitesse moyenne et la dépense, c'est-à-dire, sans perdre de vue en aucun instant le but spécial et vraiment utile de nos recherches $11^{5}$.

Le programme présenté est donc essentiellement expérimental. II vise avant tout un « but spécial et utile " : celui d'établir des tables de dépenses d'eau par des orifices qui puissent servir pour la construction et le fonctionnement des machines hydrauliques et des écluses. Si la recherche de "lois physiques ou mathématiques " est également évoquée, elle s'inscrit dans une démarche étroitement balisée, puisqu'il s'agit seulement de déterminer les causes perturbatrices expliquant l'écart entre les dépenses théoriques, telles qu'elles sont calculées avec «les formules en usage ", et les dépenses mesurées expérimentalement.

Le souci de répondre aux «besoins de la pratique » explique que ce programme d'expériences soit mené en vrai grandeur, avec un dispositif se rapprochant le plus possible d'une installation industrielle. Or, l'expérimentation en grand se distingue nécessairement de celle en petit par le nombre des participants, la nature des instruments, la précision des résultats et le mode d'exposition. Elle mobilise en général des dispositifs existants, créés dans un but utilitaire, quitte à les adapter pour les expériences : en hydraulique, cela concerne le plus souvent des canaux, des écluses, des fontaines, des bassins ou des machines hydrauliques. Sous sa forme la plus simple et la plus ancienne, l'expérimentation en grand se confond avec l'expérience au sens de simple observation raisonnée. C'est de cette manière qu'ont été établies les règles traditionnelles utilisées dans la pratique constructive. Torricelli lui-même aurait eu la première idée de sa loi en observant

5 « Expériences hydrauliques », p. 268. qu'un jet d'eau vertical s'élève presque au niveau du réservoir qui l'alimente. Les expériences faites à la fin du XVIIII siècle par du Buat sur le canal du Jard et la rivière de Hayne près de Condé-sur-l'Escaut, sont du même type'. Mais ces observations ou expériences en grand ne permettent pas d'obtenir des résultats précis, en particulier en hydraulique. D'où l'importance des expériences en petit, dans des conditions mieux contrôlées. Dès la première moitié du XVIIe siècle, des expériences sur l'écoulement par un orifice, utilisant des tuyaux et des vases, sont menées par Torricelli et Mersenne. Le grand programme de recherche en hydraulique lancé à la fin des années 1660 par l'Académie royale des sciences, sous l'égide de Huygens, Picard et surtout Mariotte, utilise également des montages expérimentaux de petite dimension. Lorsqu'en 1766, Borda reprend à son tour la question de l'écoulement des fluides par des orifices, munis ou non d'ajutages, il réalise une fois encore ses expériences avec des vases ${ }^{7}$. II en est de même, enfin, pour les expériences sur la veine liquide que Venturi mène à l'amphithéâtre de physique de Modène, au début des années 1790, et pour celles d'Hachette sur le même sujet à l'École polytechnique, en 1814 et $1815^{8}$.

Malheureusement, en hydraulique, plus encore qu'en résistance des matériaux, l'expérimentation est dominée par la question des échelles. Les phénomènes de viscosité, en particulier, dépendent de l'échelle spatiale d'observation, si bien qu'on ne peut passer sans précautions de résultats obtenus en petit à des conclusions en vraie grandeur. Si les hydrauliciens de la seconde moitié du XVIII e siècle n'étaient pas encore conscients du problème, du moins cherchèrent-ils à réaliser des expériences plus conformes aux conditions de la pratique, et donc à plus grande échelle, la difficulté étant qu'elles soient précises et bien contrôlées. Comme l'écrit l'abbé Bossut, «la simple réflexion suffit pour faire voir que dans les matières de physique, où l'on cherche seulement à découvrir la marche générale d'un phénomène sans la vouloir soumettre à la précision du calcul, les expériences doivent être faites aussi en grand qu'il est possible. Mais veut-on obtenir des résultats précis

6 P. Du Buat, Principes d'hydraulique, vérifiés par un grand nombre d'expériences faites par ordre du gouvernement, $2^{e}$ édition, Paris, 1786, tome 2, §§403-407, pp. 99-110.

7 J. C. Borda, "expériences d'écoulement dans les vases ", Mémoires de l'Académie royale des sciences (1766), 1769, p. 579-607. Sur la loi de Torricelli, voir M. Blay, La Science du mouvement des eaux de Torricelli à Lagrange, Paris, Belin, 2007. 8 G. B. Venturi, Recherches expérimentales sur le principe de la communication latérale du mouvement dans les fluides, Paris 1797, et Hachette, Traité des machines, éd. 1828, pp. 73-102. 
et destinés à jeter les fondements d'une théorie ? Les expériences fort en grand n'ont plus le même avantage. Presque jamais elles ne peuvent être assez exactes, assez répétées, assez variées, pour faire disparaître les différences sensibles qui se trouvent entre plusieurs observations semblables. Entre ces deux écueils, il y a un milieu à prendre. Opérer assez en grand pour rendre les effets distincts, sans sortir des bornes compatibles avec la précision : voilà le principe qui doit diriger les expériences auxquelles on veut appliquer la Géométrie " ${ }^{9}$.

De fait, dans ses expériences, qui servent de références pendant un demi-siècle, Bossut adopte une solution intermédiaire : après avoir utilisé les fontaines de Mézières, il expérimente sur la résistance des fluides avec des modèles de vaisseaux évoluant dans un bassin de l'École militaire de Paris. Dans ses recherches sur la résistance des fluides à l'écoulement, financées par le ministère de la guerre, au début des années 1780, l'ingénieur du Buat expérimente lui aussi sur des dispositifs de taille moyenne, utilisant un canal factice fait de madriers de chêne. Les expériences sur l'écoulement par des orifices réalisées au Piémont par Francisco Domenico Michelotti sont d'un ordre de grandeur comparable. À son instigation, le roi de Sardaigne Charles-Emmanuel III a fondé en 1763 un établissement pour les expérimentations hydrauliques, situé au lieu-dit de La Parella près de Turin. L'installation comprend un canal alimenté par la Doire, une tour carée de huit mètres de haut, qui sert de château d'eau, et deux réservoirs en contrebas. Sur l'une des faces de la tour trois orifices à différentes hauteurs permettent des expériences sur les dépenses d'eau. Les réservoirs servant de jauge recueillent l'eau qui se déverse des orifices. Les expériences de Michelotti sont poursuivies et complétées par ses fils Giuseppe et lgnazi10. Giorgio Bidone, formé par lgnazio, reprend la direction de l'établissement de La Parella et y mène d'importantes expériences sur les dépenses d'eau et la contraction de la veine liquide en 1819 et en 1827-1828 ${ }^{11}$.

9 J. Le Rond d'Alembert, N. Caritat de Condorcet et Ch. Bossut, Nouvelles expériences sur la résistance des fluides, Paris, Jombert. 1777, pp. 5-6. Le rédacteur est Bossut.

10 F. D. Michelotti, Sperimenti idraulici diretti principalmente a confermare la teoria e facilitare la pratica del misurare le acque correnti, Turin, 2 vol., 1767-1771, et G. T. Michelotti, " Mémoire physico-mathématique contenant les résultats des expériences hydrauliques faites près de Turin en 1783, 11 in Mémoires de l'Académie royale des sciences, années 17841785, 1786. Sur l'installation de la Parella, voir A. C. Scolari, « La torre per gli esperimenti idraulici di Francesco Domenico Michelotti I, L'ambiente storico, 6/7 (1983-1984), pp. 62-90, et la note d'Hachette dans son Traité de mécanique, édition de 1828, p. 255.

11 G. Bidone, «Expériences sur divers cas de la contraction de la veine fluide et remarques sur la manière d'avoir égard
Comme les autres, les expériences de La Parella utilisent des dispositifs ad hoc assez éloignés de ceux que l'on trouve dans la pratique. L'alimentation s'y fait directement par un réservoir et les orifices sont petits, le plus souvent carrés et à bords uniformes. Dans les usines et les écluses, en revanche, des canaux d'arrivée amènent l'eau par filets horizontaux. Les orifices euxmêmes sont souvent assez larges, rectangulaires et pourvus à leur côté supérieur d'une vanne mince que l'on peut lever et abaisser à volonté. Certes, des expériences effectuées sur des écluses de moulins et de canaux ont été réalisées en 1782 par l'ingénieur du canal du Midi Lespinasse, mais elles demeurent parcellaires et trop imprécises ${ }^{12}$. C'est pour pallier ces manques que Poncelet et Lesbros entreprennent de nouvelles expériences plus systématiques et plus proches des conditions de la pratique.

\section{Entre théorie et expérience}

Avantd'examinerle travaildes deuxexpérimentateurs, il reste à préciser le problème des dépenses d'eau (ou débits) ${ }^{13}$. Etudier l'écoulement de l'eau par un orifice est une question à la fois théorique et expérimentale. II s'agit principalement de déterminer la vitesse de l'eau à la sortie en fonction des caractéristiques de

à la contraction dans le calcul de la dépense des orifices ", Memorie della reale Accademia delle scienze di Torino, tome 27, 1823, pp. 83-136, « Expériences sur la dépense des déversoirs et sur l'accélération et la courbure qu'ils occasionnent à la surface du courant ॥, ibid., t. 28 (1824), p. 281-330, et « Esperienze sulle contrazioni parziali delle vene d'acqua ". Memorie della Societa Italiana delle Scienze, t. 20 (1828), pp. 536-572. Bidone a publié par la suite d'autres mémoires sur le même sujet.

12 G.-C. Lespinasse, "Mémoire concernant les dépenses d'eau des grands réservoirs par des orifices considérables, accompagné de diverses expériences propres à indiquer la cause et la mesure du déchet que ces dépenses éprouvent, les réservoirs étant constamment entretenus pleins ", Académie des sciences, inscriptions et belles-lettres de Toulouse, † . 2, 1784, pp. 39-70. Le mémoire, dont une copie manuscrite est conservée dans le fonds Poncelet de l'École polytechnique (AEP, IX PO 7/140), a été lu très attentivement par Poncelet et Lesbros (op. cit., §14, pp. 264-265). Ils connaissent également les expériences en vraie grandeur faites par Kypke près du canal de Bromberg en 1799 et rapportées par Eytelwein (voir J. A. Eytelwein, Handbuch der Mechanik fester Körper und der Hydraulik mit vorzüglicher Rücksicht auf ihre Anwendung in der Architektur, Berlin, 1801, §104, pp. 136-140, et J. V. Poncelet et J. A. Lesbros, op. cit., $\S 15$, p. 266).

13 Voir M. Blay, La science du mouvement des eaux de Torricelli à Lagrange, Paris, Belin, 2007, et J. S. Calero, The Genesis of Fluid Mechanics, 1640-1780, Springer, 2008, pp. 271-352. 
la dépense, c'est-à-dire du volume de liquide sortant de l'orifice par unité de temps, et de l'alimentation : taille, forme et position de l'orifice, charge (hauteur d'eau au-dessus du centre de l'orifice) et dimension du canal d'amenée ou du réservoir. Accessoirement, il s'agit aussi de préciser la nature de l'écoulement et la forme de la veine liquide. Si les fontainiers ont développé depuis longtemps des savoirs et des savoir faire sur les dépenses d'eau, dont on trouve par exemple des indices dès l'Antiquité dans le livre de Frontin, ils ne disposent d'aucune loi sur l'écoulement des fluides par un orifice, sinon, peut-être, que la dépense est toujours proportionnelle à la section de l'orifice, toutes choses égales par ailleurs.

Il faut attendre 1644 pour qu'une véritable loi soit énoncée par Torricelli. Ce disciple de Galilée suppose que la vitesse de l'eau sortant d'un réservoir est égale à celle d'un corps tombant d'une hauteur égale à la charge. Si $v$ est la vitesse de l'eau à la sortie, $h$ la charge et $g$ l'accélération de la pesanteur, on obtient la formule $v=\sqrt{2 g h}$ en appliquant la loi de la chute des corps. Connaissant l'aire de l'orifice $S$, on déduit alors la dépense, égale à $S \sqrt{2 g h}$. Mersenne et Descartes parviennent à peu près au même moment à des résultats identiques. La formule de Torricelli paraît d'ailleurs confirmée par l'expérience : si on oriente le jet sortant d'un orifice vers la verticale au moyen d'un ajutage coudé, on constate qu'il s'élève à peu près jusqu'au niveau du réservoir.

En fait, le jet vertical n'atteint jamais tout à fait ce niveau. Torricelli, qui a noté ce phénomène, l'attribue seulement à la résistance de l'air. D'autres auteurs, en revanche, sont amenés à revoir sa formule. Ainsi, en faisant varier la taille de l'orifice et la forme de l'ajutage, Mariotte arrive à la conclusion que le frottement du fluide sur les parois diminue la vitesse à la sortie ${ }^{14}$. De son côté, Newton observe que la veine liquide se rétrécit peu après la sortie selon la proportion $S^{\prime} / S=1 / \sqrt{2}$ ( $S$ est la section de l'orifice et $S$ ' celle de la veine contractée; le rapport S'/S est appelé par les auteurs du XIXe siècle le coefficient de contraction). Or, $v$ étant la vitesse à l'orifice et $v$ ' la vitesse au niveau de la veine contractée, la loi de continuité du fluide donne l'égalité $S v=S$ ' $v$ ' et par conséquent $v=v^{\prime} / \sqrt{2}{ }^{15}$. Newton suppose que la

14 E. Mariotte, Traité du mouvement des eaux et des autres corps fluides, Paris, 1686. Sur les travaux des académciens, voir M. Blay, op. cit.

15 La loi de continuité pour un écoulement stationnaire d'un fluide incompressible semble avoir été connue depuis longtemps des ingénieurs hydrauliciens. On la trouve énoncée par Léonard de Vinci à propos de l'écoulement uniforme d'une rivière. Voir P. Duhem, Études sur Léonard de Vinci. Ceux qu'il valeur de v' est donnée par la formule de Torricelli et il en déduit que la vitesse $v$ à l'orifice se réduit à $\sqrt{g h}$, soit la valeur théorique que donnerait une charge égale à seulement la moitié de la charge effective. Selon lui, s'il y a perte de vitesse à l'orifice, et donc de charge, c'est parce que le fluide, au voisinage de l'orifice, ne s'écoule pas par filets parallèles. Des filets obliques, près des bords de l'orifice, ralentiraient l'écoulement général jusqu'au niveau de la veine contractée, où la vitesse redeviendrait uniforme dans toute la section ${ }^{16}$. Dans son Hydrodynamica, publiée en 1734, Bernoulli déduit la formule de Torricelli du principe des forces vives ${ }^{17}$. Sa démonstration suppose un écoulement du fluide par tranches parallèles; or, remarque-t-il, l'écoulement est fortement perturbé au niveau de l'orifice et ne redevient régulier qu'à celui de la veine contractée. C'est pourquoi, pour Bernoulli comme pour Newton, la formule de Torricelli ne fournit la vitesse de l'eau qu'après la contraction de la veine et non à l'orifice de sortie.

Tous les hydrauliciens sont désormais d'accord pour introduire un coefficient correcteur dans le calcul de la vitesse à l'orifice, mais sans s'accorder sur sa valeur. Newton l'estime, comme on l'a vu, à $1 / \sqrt{2} \approx 0,71$; les expériences menées par Bossut, Michelotti, Hachette, Bidone et d'autres, montrent non seulement que ce coefficient est en général trop grand mais qu'il varie fortement en fonction des conditions de la dépense (taille, forme, charge, etc.). Résumant les incertitudes, Poncelet et Lesbros notent dans leur mémoire que pour des orifices circulaires les coefficients peuvent varier en fonction de la taille de 0,60 (diamètre supérieure à $2 \mathrm{~cm}$ ) à 0,78 (diamètre inférieure à $1 \mathrm{~mm}$ ) et que les variations en fonction de la charge sont probablement aussi grandes, bien que moins connues.

Il est évidemment impossible de mesurer directement la vitesse de l'eau à l'orifice. Comme l'explique Borda, les expérimentateurs peuvent utiliser deux méthodes pour y parvenir. La première, utilisée par Newton, consiste à déduire la vitesse à l'orifice $v$ de la vitesse théorique de sortie, en tenant compte la contraction de la veine. En notant $\mu$ le coefficient de contraction S'/S (voir plus haut), la vitesse à l'orifice

a lus et ceux qui l'ont lu, le série, Paris, 1906, pp. 195-198.

16 I. Newton, Principia, proposition 36. Newton a revu entièrement le traitement du problème dans la seconde édition des Principia, en 1713. Voir G. E. Smith, "Newton's study of fluid mechanics", International Journal of Engineering Science, vol. 36, 1998, pp. 1377-1390, et J. S. Calero, op. cit., pp. 95-102 et pp. 282-283.

17 D. Bernoulli, Hydrodynamica, sive de viribus et motibus fluidorum commentarii, Strasbourg, 1738, p. 38. 
s'écrit alors $\mu \sqrt{2 g h}$. Comme il est très difficile de mesurer le diamètre de la veine contractée et plus encore sa section, la méthode est peu praticable. La seconde, utilisée par Mariotte et la plupart des expérimentateurs, consiste à mesurer la quantité d'eau $Q$ dépensée pendant un temps donné T. Si S est l'aire de l'orifice, la vitesse $v$ de l'eau est alors égale à $Q / S T$. L'expérience montre que cette vitesse diffère de la vitesse théorique $\sqrt{2 g h}$. Si l'on admet alors, comme le font les hydrauliciens, que le coefficient de correction $v / \sqrt{2 g h}$ est égal au coefficient de contraction $\mu$, les deux méthodes sont équivalentes. Cela suppose cependant l'hypothèse du parallélisme des tranches pour la veine contractée, ce qui n'est en rien démontré.

Les mesures montrent que la valeur du rapport $v / \sqrt{2 g h}$ dépend en fait largement des conditions d'expérience. C'est pourquoi Poncelet et Lesbros adoptent une position très prudente : " La détermination du coefficient de contraction relatif aux divers orifices, ou, pour parler plus exactement, la détermination du coefficient de correction, de réduction, par lequel il convient de multiplier, dans chaque cas, la formule théorique de la dépense d'un orifice, pour obtenir la dépense vraie et observée, cette détermination, disons-nous, qui, à la première vue, paraît être la chose la plus facile à obtenir par le moyen de l'expérience, est, par le fait même de sa variation dans les divers cas, celle qui présente le plus de difficultés, ou plutôt cette détermination comprend à elle seule la solution de presque toutes les lois du mouvement des fluides; car, si la valeur du coefficient d'une même formule doit varier par le changement seul des quantités dont elle est fonction, c'est un signe certain que cette formule ne satisfait point aux expériences, et qu'elle peut tout au plus être considérée comme le premier terme de la fonction rigoureuse dont elle offre une valeur plus ou moins approchée dans certaines circonstances $11^{18}$.

Cette réflexion est la clé du travail expérimental de Poncelet et Lesbros. Pour eux, la formule de Torricelli n'exprime pas une loi physique, c'est tout au plus une référence commode. La réalité physique se cache plutôt dans le coefficient de correction et elle est hors de portée de la théorie, au moins dans l'immédiat. Cela a deux conséquences : d'abord, le problème de la dépense d'eau n'est plus une question vive pour I'hydrodynamique. Ceci est vrai, en fait, dès la fin du XVIIIe siècle, quand les recherches expérimentales les plus fécondes pour le développement de la discipline portent sur l'écoulement dans les canaux

18 « Expériences hydrauliques », p. 253. et les tuyaux et non, comme autrefois, sur celle par les orifices. Les phénomènes résultant de la viscosité du liquide et du caractère turbulent de son écoulement sont en effet mieux analysables dans le premier cas du fait que le contact avec la paroi y est continu, que dans le second où la discontinuité à l'orifice introduit des effets d'une grande complexité dépendant de la configuration. Ensuite, le statut même de l'expérience dans le cas des dépenses d'eau s'en trouve modifié. La motivation se déplace de la théorie des fluides à l'hydraulique pratique. L'objectif principal n'est plus d'étudier expérimentalement les lois de l'écoulement du fluide mais de fournir directement aux praticiens des informations pour la construction de dispositifs spécifiques comme des pertuis d'écluses et d'usines. Dans ces conditions, le coefficient de contraction, présenté sous forme tabulée, devient lui-même un module sans signification physique, servant seulement aux constructeurs pour le calcul des dépenses d'eau de leurs installations.

Il est intéressant de comparer cette approche à celle adoptée par Poncelet pour la réalisation de sa fameuse rove à aubes courbes quelques années plus tôt. Poncelet a conçu cette machine à partir d'une démarche purement théorique, afin d'illustrer le principe des forces vives. Pour une certaine vitesse de la roue, le mouvement de l'eau y satisfait à deux conditions, l'absence de chocs à l'entrée et la vitesse nulle à la sortie, de sorte que la force vive est intégralement transmise à la machine. Le rendement théorique maximal est donc de $100 \%$. Dans la réalité, il est nettement plus faible, entre $57 \%$ et $67 \%$ environ selon le débit de l'eau. La différence s'explique principalement par le caractère turbulent de l'écoulement. Poncelet a entrepris une série d'expériences pour fixer les coefficients correcteurs permettant de passer du rendement théorique au rendement réel. En apparence, la démarche expérimentale est donc la même qu'avec les dépenses d'eau, où il s'agit également de corriger une formule théorique déduite du principe des forces vives, la formule de Torricelli. Mais on notera immédiatement la différence : dans le premier cas, on part de principes dont la validité n'est pas contestée, en en corrigeant les conséquences pour tenir compte des circonstances particulières; dans le second, on part d'une loi établie à partir d'hypothèses contraires à la réalité physique (le parallélisme des tranches) et on utilise des coefficients correcteurs pour l'ajuster aux résultats fournis par l'expérience. En somme, on passe d'une approche expérimentale visant à compléter et à corriger la théorie à une approche expérimentale visant à décrire un comportement 
physique de manière purement phénoménologique et hors de toute considération théorique.

\section{L'organisation des expériences}

C'est en 1827 que Poncelet et Lesbros engagent leurs expériences sur les dépenses d'eau. Le directeur de l'École de l'artillerie et du génie a adressé en juin une note rédigée par Poncelet et Lesbros au Ministre de la guerre. Le président du comité des fortifications, Rognat, a apporté son soutien au projet. L'autorisation arrive, avec les fonds, en août. La construction des appareils commence en septembre. À la fin novembre, Poncelet et Lesbros entreprennent une première série d'expériences. Elle dure pendant plus d'un mois. À cette date, Poncelet et Lesbros ont déjà mené ensemble plusieurs expériences du même type dans le cadre de recherches sur les roves hydrauliques à aubes courbes. Pour construire une machine hydraulique et évaluer sa puissance, il faut en effet connaître la puissance disponible et celleci dépend directement de la dépense d'eau. Ainsi, Poncelet, aidé de Lesbros, a effectué dès 1824 des mesures sur l'écoulement par un orifice rectangulaire de petite taille. L'année suivante, Poncelet a mesuré des dépenses sur les moulins de la ville de Metz. Les expériences hydrauliques engagées en 1827 sont la suite directe de ces premiers travaux ${ }^{19}$. Au début de l'année 1828, Poncelet et Lesbros adressent une deuxième note au Ministère de la guerre, rendant compte des opérations de leur campagne précédente et de celles qui restent à entreprendre. Lesbros reprend les expériences en août 1828, grâce à une nouvelle subvention, et les poursuit en 1829. Un premier mémoire, présenté par Poncelet à l'Académie des sciences le 16 novembre 1829, est publié en 1832 dans la série des Savants Etrangers ${ }^{20}$. ॥ porte sur les expériences de 1827 et 1828 relatives aux orifices rectangulaires en mince paroi de $20 \mathrm{~cm}$ de base, complètement isolés des faces latérales et du fond du réservoir. Poncelet ayant renoncé, Lesbros termine seul l'entreprise dans les années suivantes. Les résultats de ses expériences sont publiés après un long délai, en 1852.

19 Voir B. Belhoste et L. Lemaître, « J.-V. Poncelet, les ingénieurs militaires et les roves et turbines hydrauliques $॥$, dans Le Moteur hydraulique en France au XIXe siècle : concepteurs, inventeurs et constructeurs, Cahier d'histoire des sciences et des techniques, n² 29, 1990, pp. 33-89.

20 Le mémoire, présenté par Poncelet à l'Académie des sciences le 16 novembre 1829 (Procès verbaux des séances de l'Académie des sciences tenues depuis la fondation de I'Institut jusqu'au mois d'août 1835, †. 9, p. 358), est approuvé sur un rapport de Girard le 2 mai 1831 (ibid., pp. 622-628).
Nous nous intéresserons seulement ici aux expériences publiées dans le premier mémoire, signé conjointement par Poncelet et Lesbros (dans cet ordre). Poncelet en est le concepteur et le rédacteur. Tombé malade, il a du laisser la conduite des expériences à Lesbros. C'est donc ce dernier qui tient le registre-journal, réalise les calculs préliminaires et dresse les tables détaillées jointes au mémoire. Poncelet se plaît à souligner les qualités d'expérimentateur de son collaborateur, «l'habileté qu'il a acquise dans les opérations de ce genre, ses connaissances en hydraulique, et, par-dessus tout, son activité infatigable, son exactitude scrupuleuse dans les observations et les calculs $11^{21}$. Cependant, les deux hommes n'auraient pu réaliser une tâche aussi délicate et complexe sans l'aide d'assistants dévoués. L'appareil d'expérience et les instruments ont été construits dans les ateliers de l'École de Metz par Bodin et Faivre, sous la direction des mécaniciens Aimé et Savart22. Dans la réalisation des expériences, Lesbros a été aidé par le garde du génie Schuster, un employé de l'École entraîné aux observations de précision ${ }^{23}$, ainsi que par le sergent au 3e régiment du génie Mangonnet et par le maître-ouvrier au même régiment Grandidier (partis tous deux en Morée en octobre 1828, ils sont remplacés par l'ouvrier en bois

21 «Expériences hydrauliques 》, avant-propos, pp. 243-244, où Poncelet précise la part de chacun.

22 L'artiste mécanicien Louis François Marcel Bodin (1798-1873), ancien élève de l'École des arts et métiers de Châlons, est employé à l'atelier de l'École de l'artillerie et du génie, à la tête duquel il succède à Savart en 1843. Sur Bodin, voir la notice nécrologique rédigée par dans les Mémoires de l'Académie de Metz, 56 année, 1874-1875, p. 393-405. Faivre, adjoint d'Aimé, semble avoir quitté l'École de l'artillerie et du génie pour raisons de santé au début des années 1830. Quant aux gardes du génie François Sébastien Aimé (1762-1843) et Gérard Savart (1759-1843), ils sont deux anciens artistes de Mézières, tous deux protégés de Monge. Aimé a fait partie de l'Expédition d'Égypte. Savart est le père du physicien Félix Savart. À l'École d'artillerie et du génie de Metz, ils sont responsables de l'atelier et de la galerie des modèles de l'École d'artillerie et du génie de Metz. L'un et l'autre ont été membres de l'Académie de Metz. Sur Savart et ses enfants, voir la notice rédigée par F. Blanc dans les Mémoires de l'Académie de Metz, 36e année, 1854-1855, pp. 585-596.

23 Le garde du génie Jean-Jacques Schuster (1797-1853) a été employé à l'École de l'artillerie et du génie où il a terminé sa carrière comme chef d'administration. Il est connu pour ses observations météorologiques commencées en 1825 à l'invitation de François Arago. Il a été membre de l'Académie de Metz. Voir sa notice nécrologique rédigée par E. Grelois dans les Mémoires de l'Académie de Metz, 34e année, 1852-1853, pp. 87-89. 
de l'arsenal du génie Pistre). Poncelet n'oublie pas de rendre hommage à ces " techniciens invisibles» : " L'intelligence de ces hommes, leur zèle, leur dévouement même, car il en faut beaucoup pour assujettir son esprit et son corps à des fonctions aussi minutieuses, sont dignes des plus grands éloges $12^{24}$.

La réalisation des expériences en vraie grandeur exige un dispositif complexe. II faut un bassin de retenue maintenu toujours à niveau et un bassin de jauge, pour mesurer les quantités dépensées. Les expériences ont lieu dans l'enceinte des fortifications de la ville de Metz, qui offre des canaux et des plans d'eau (voir fig. 1). Le dispositif général consiste à réunir dans un réservoir les eaux de la Moselle supérieure et à les décharger dans la rivière plus en aval, après qu'elles aient passé par un appareil d'expérience. Cet appareil est installé juste en amont du pont du Pontiffroy ( $\left.n^{\circ} 4\right)$, à la sortie de la ville, et à $90 \mathrm{~m}$ à l'ouest des fossés du front de Saint-Vincent $\left(n^{\circ} 3\right)$. Ces fossés qui fournissent l'eau au réservoir sont alimentés par la Moselle à $1100 \mathrm{~m}$ en amont $\left(n^{\circ} 5\right)$. Entre la vanne d'admission et l'embouchure du canal de décharge, en aval de l'appareil d'expérience, le dénivelé atteint $4 \mathrm{~m}$. En fait, la charge maximale réellement disponible ne dépasse pas $2 \mathrm{~m}$ pour diverses raisons (principalement la présence du bassin de jauge et la hauteur de l'orifice au-dessus du fond).

Examinons de plus près le dispositif général (fig. 2). Le niveau de l'eau dans les fossés du front Saint-Vincent, formant un bassin de $25000 \mathrm{~m}^{2}$, est réglé en amont par la vanne d'admission. L'alimentation des fossés du

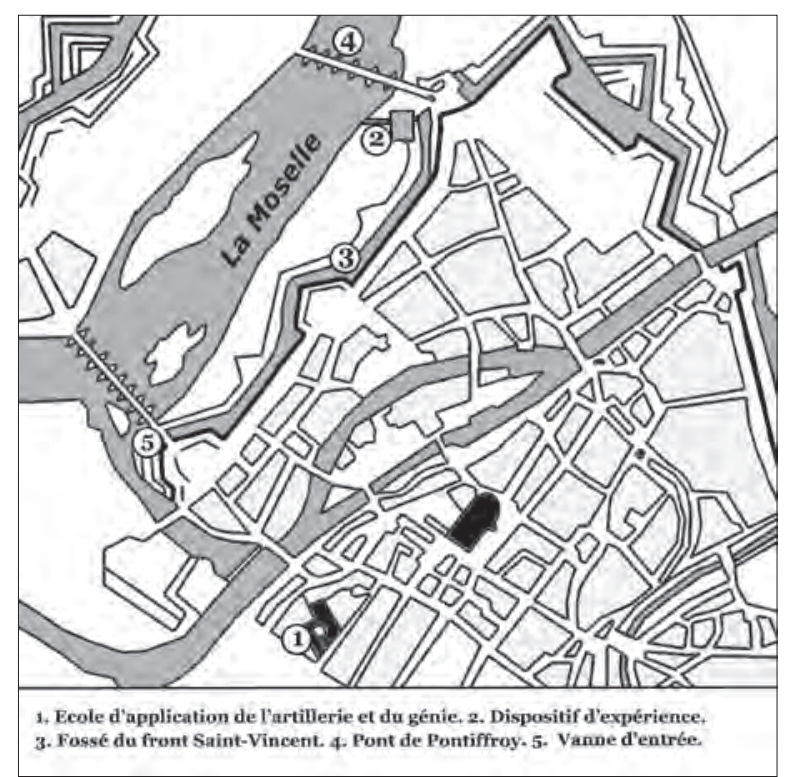

fig. 1 : Metz en 1827

front de Chambière, séparé de ceux du front de SaintVincent par un batardeau, y provoque des irrégularités importantes, ce qui oblige Poncelet et Lesbros à faire aménager un réservoir de $1500 \mathrm{~m}^{2}$ relié aux fossés par un canal. La présence d'excavations anciennes a considérablement facilité les travaux, qui se sont réduits à la construction d'une digue en travers. Une vanne de garde sur le canal d'amenée et une vanne de décharge permettent de régler à volonté le niveau du réservoir. À ce réservoir est adjoint un bassin de

fig. 2 : Le dispositif général

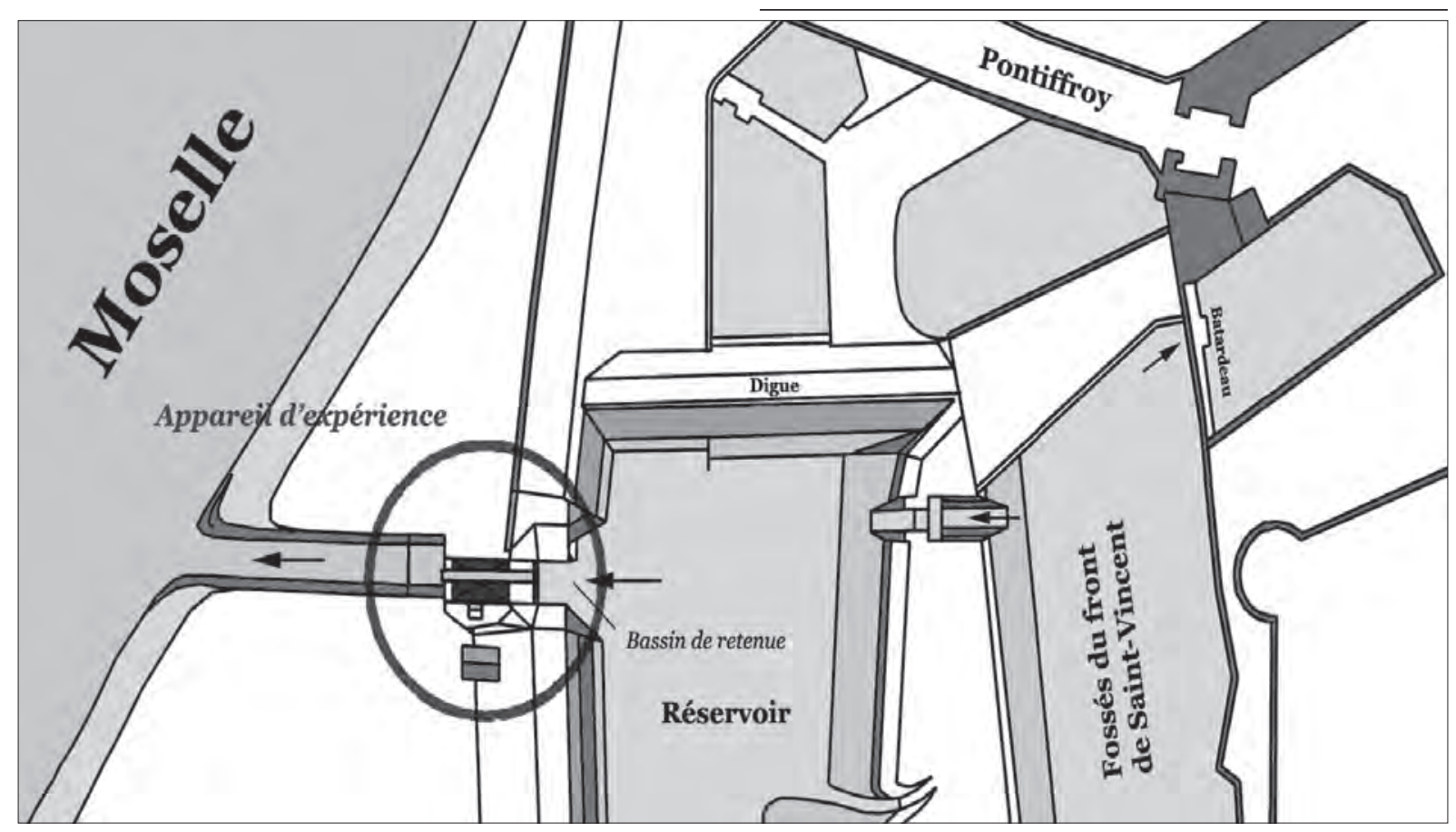


retenue de 3,68 $\mathrm{m}$ de large et de $3 \mathrm{~m}$ de profondeur, qui alimente directement l'appareil d'expérience.

Cet appareil comprend lui-même un barrage avec un orifice muni d'une vanne, un canal de conduite à la sortie et un bassin de jauge (voir fig. 3). Le barrage est formé d'un châssis de madriers de $5 \mathrm{~cm}$ d'épaisseur fixé sur les montants du bassin de retenue. L'orifice, à minces parois et de forme rectangulaire, se trouve en son milieu, à 1,74 $\mathrm{m}$ des faces latérales du bassin. Sa base est placée à environ $54 \mathrm{~cm}$ du fond, ce qui donne une charge disponible de 2 mètres en 1827 et de $1,60 \mathrm{~m}$ en 1828. Sa largeur est de $20 \mathrm{~cm}$ et sa longueur verticale varie selon la hauteur d'une vanne en cuivre de $4 \mathrm{~mm}$ d'épaisseur. Pour une ouverture maximale, correspondant à une aire de $0,25 \mathrm{~m}^{2}$, il débite jusqu'à $1 \mathrm{~m}^{3} / \mathrm{s}$ d'eau sous une charge de $2 \mathrm{~m}$. À l'origine, une lame de cuivre mince terminée par un biseau garnit son bord inférieur et supérieur. Une déformation de plus de 1/300 ayant été constatée au terme de la première campagne d'expérience, Poncelet et Lesbros renforcent les minces parois en faisant construire dans les ateliers de l'École d'application une plaque de cuivre de $4 \mathrm{~mm}$ d'épaisseur dans laquelle est pratiquée une ouverture de $20 \mathrm{~cm}$. À la sortie de l'orifice, l'eau est recueillie dans un canal de conduite, en madriers de sapin, de $60 \mathrm{~cm}$ de large et de $11 \mathrm{~m}$ de long, calfaté ou mastiqué sur toute sa longueur, et débouchant sur le canal de décharge relié à la Moselle. Le bassin de jauge est situé sous le canal de conduite. D'une capacité maximale de $24 \mathrm{~m}^{3}$, il a $6 \mathrm{~m}$ de longueur, $4 \mathrm{~m}$ de largeur et $1 \mathrm{~m}$ de profondeur. Il est revêtu de madriers soigneusement calfatés. Un puits d'observation servant à la mesure des hauteurs d'eau communique avec le bassin.

La construction, faite très rapidement, est imparfaite. Poncelet et Lesbros constatent qu'il aurait été bien préférable de réaliser le bassin de jauge en maçonnerie. Les problèmes d'étanchéité rendent les opérations très difficiles pendant la première campagne d'expériences. Un incident abîme le fond et gêne l'évacuation du bassin de jauge. "L'événement nous a prouvé, comme dans toutes les occasions semblables, qu'on ne gagne rien à précipiter les choses $11 .{ }^{25}$ Mais il faut aller vite et obtenir des résultats utiles aux élèves pour justifier les fonds. Les joints s'étant bouchés avec le temps, les conditions s'améliorent sensiblement en 1828, ce qui permet à Poncelet et Lesbros de refaire les expériences de l'année précédente.

25 «Expériences hydrauliques », p. 280.
Des opérations préliminaires aux résultats des mesures Les expériences consistent essentiellement à mesurer les quantités d'eau dépensées dans des conditions données (taille de l'orifice, charge). Elles sont effectuées dans le bassin de jauge, qui récupère l'eau dépensée, en mesurant le niveau. Si on connaît la surface du bassin, le volume s'en déduit directement. En fait, la méthode est vicieuse, car il est très difficile de faire des bassins réguliers. ॥ faut donc préalablement étalonner le bassin de jauge, comme l'avait déjà fait Bossut, en versant des quantités d'eau connues et en mesurant la hauteur correspondante. L'opération exige un module. Le premier module utilisé, une caisse en bois de $50 \mathrm{~cm}$ de côté, s'avère trop imprécis et Poncelet et Lesbros doivent faire appel aux ressources de l'École d'application. La caisse est alors remplacée par un décalitre cylindrique en cuivre fort du cabinet des modèles, construit par Merklein, dont la contenance à été vérifié dans l'atelier de précision. Pour enlever la couche d'eau en excès les expérimentateurs utilisent une glace de cristal épaisse appartenant à la machine électrique de l'École. L'eau est alors versée, à la manière de Bossut, dans un tonneau bien étalonné de 968 litres servant de jauge intermédiaire.

II reste à vidanger les tonneaux dans le bassin de jauge pour l'étalonner. L'opération est très délicate. La hauteur de l'eau est mesurée après chaque vidange au moyen d'une verge munie d'un curseur avec vernier (mesure au $10^{e}$ de $\mathrm{mm}$ ). L'agitation du bassin ne permet pas d'atteindre une précision supérieure au $5^{e}$ de $\mathrm{mm}$, correspondant à 5 litres d'eau. En revanche, Poncelet et Lesbros prennent en compte les pertes dues aux filtrations et à l'évaporation au cours des vidanges. Ces pertes sont d'autant plus importantes que chaque vidange prend un temps considérable. Voilà comment ils procèdent : la baisse du niveau entre deux vidanges successives est mesurée précisément. Sachant que cet intervalle dure 6 minutes, ils calculent pour chaque hauteur la baisse du niveau en 1 minute, ce qui leur permet de corriger les mesures après chaque vidange. Ayant obtenu les hauteurs occupées par les multiples de 968 litres d'eau, Poncelet et Lesbros en déduisent par interpolation le nombre de litres répondant à chaque centimètre. Enfin, ils dressent une table des volumes absolus d'eau pour chaque indication de l'échelle, en tenant compte du premier versement qui élimine l'irrégularité du fond.

Les pertes dues aux filtrations ayant été réduites au printemps 1828, Poncelet et Lesbros recommencent l'étalonnage du bassin de jauge, cette fois avec un tonneau de 1112 litres. Ils rencontrent des problèmes 


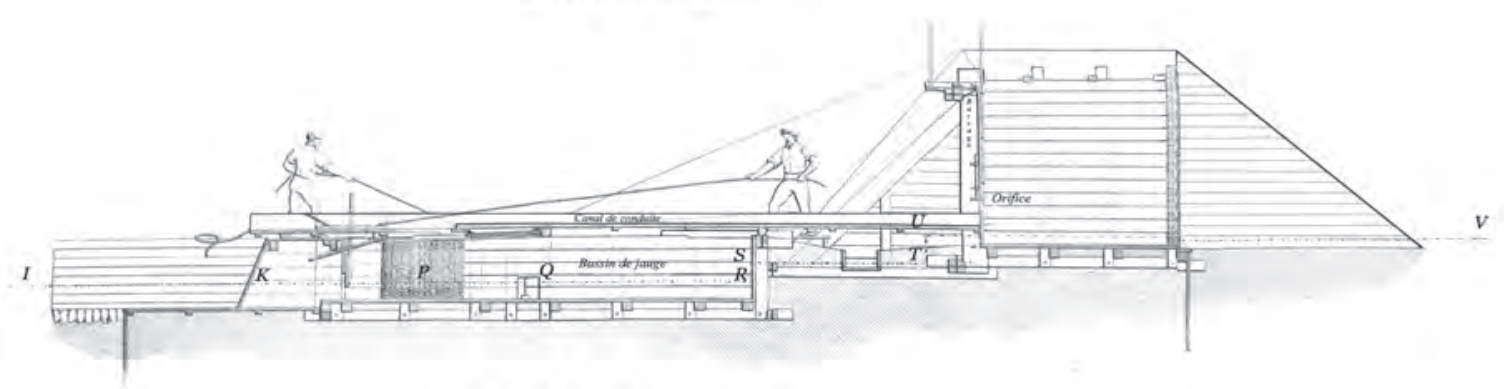

Plan suivant IKPQRSTUV

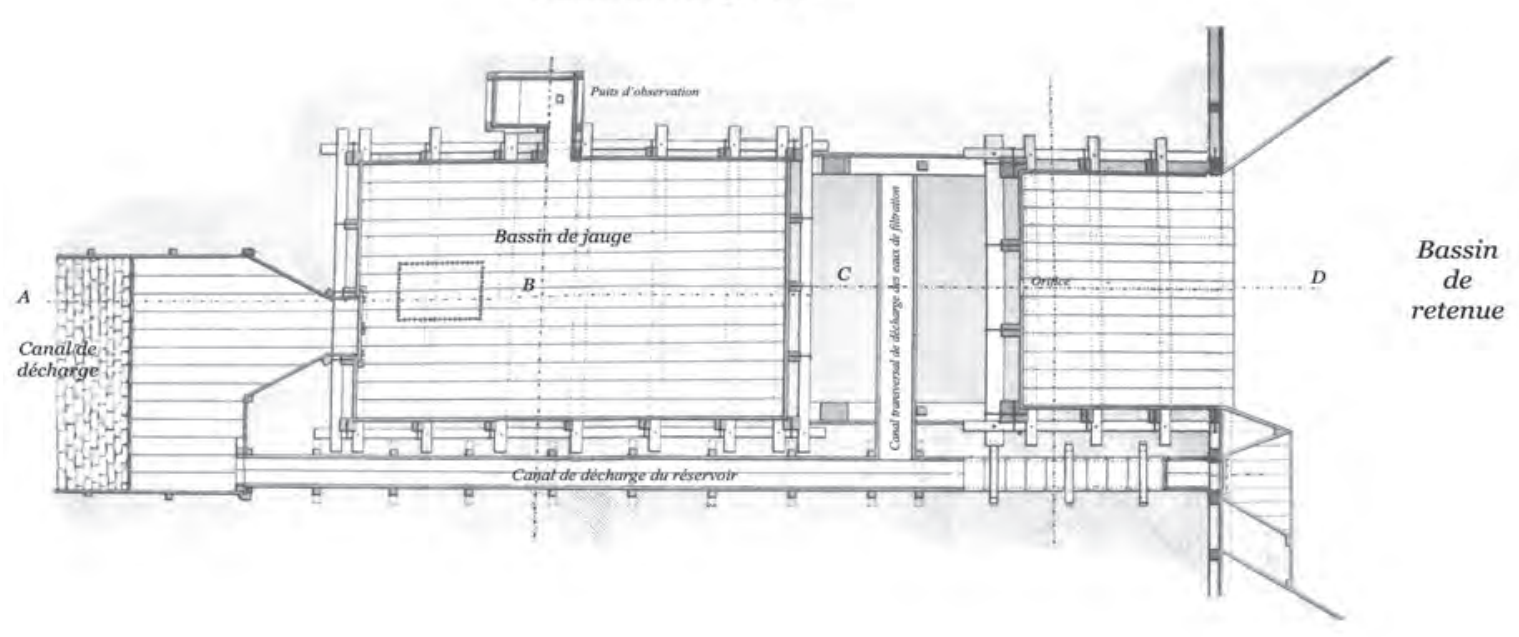

fig. 3 : L'appareil d'expérience

pour les parties basses de l'échelle de graduation. N'ayant pu en trouver la cause, ils décident de verser 8100 litres d'un coup, atteignant ainsi une hauteur de $35 \mathrm{~cm}$, qui sert de base à la table générale et définitive du jaugeage. Pour améliorer la précision dans le cas de petites dépenses, inférieure à 13 litres/s, ils remplacent le bassin de jauge par des cuviers en tronc de cône très exactement étalonnés. Toutes ces opérations préliminaires absorbent un temps considérable. Poncelet et Lesbros en soulignent néanmoins les avantages, celles « d'exercer les aides, et de donner à l'observateur lui-même une habileté et une intelligence des moindres inconvénients de l'appareil, qu'il n'eût acquises qu'aux dépens de l'exactitude dans les résultats essentiels $12^{26}$.

Outre l'étalonnage du bassin de jauge, les expérimentateurs doivent régler au préalable l'ouverture de l'orifice et la hauteur de l'eau dans le réservoir. Si la largeur de l'orifice reste fixe, sa longueur verticale varie en fonction de la hauteur de la vanne. Le réglage de cette vanne est délicat à cause de l'influence des variations

26 Ibid., p. 299. de température et d'hygrométrie. L'échelle des hauteurs a été isolée pour supprimer les effets de l'humidité des madriers. Des correctifs ont été introduits en 1828 pour tenir compte de la dilatation de la tige de manœuvre de la vanne, selon la température, et l'ensemble du dispositif a été sensiblement amélioré dans les expériences de 1829. La hauteur d'eau dans le réservoir est réglée par les vannes d'alimentation et de décharge et leurs manœuvres ne présentent pas de difficulté particulière. En revanche, la mesure de la charge exige des préparatifs, car la surface du bassin de retenue se déprime légèrement au-dessus et en avant de l'orifice. Poncelet et Lesbros décident d'effectuer au préalable deux mesures simultanées pour diverses hauteurs d'eau, l'une au-dessus de l'orifice, là où la dépression est la plus forte, l'autre, correspondant à la charge effective, à une distance suffisante pour que le fluide soit considéré comme stagnant, c'est-à-dire à environ $3 \mathrm{~m} 50$ de l'orifice. Cette dernière mesure se fait à l'aide d'une règle en bois de $3 \mathrm{~m}$, comparable à celle utilisée dans les opérations de nivellement, et munie de deux coulisseaux. Ils tirent de ce travail 
une "table de dépression ", qui leur permet de corriger les mesures prises au cours des expériences directement au-dessus de l'orifice.

Une fois ces opérations préliminaires achevées, il ne reste plus qu'à mesurer les dépenses. Pour chaque hauteur de charge, Lesbros et ses assistants varient la longueur de l'orifice depuis $5 \mathrm{~mm}$ jusqu'à $20 \mathrm{~cm}$ en réglant la vanne et mesurent à chaque fois la quantité d'eau dépensée pendant un temps donné. Une fois le bassin correctement étalonné, le jaugeage ne présente pas de difficultés majeures. La mesure du temps exige plus d'attention. Lesbros, qui réalise les expériences, utilise un chronomètre à repos de Bréguet marquant les $1 / 2$ secondes en 1827 et un chronomètre à plume marquant les $1 / 10^{e}$ de seconde, toujours de Breguet, en 1828 et 1829, ce qui permet de réduire les volumes d'eau. Le plus délicat est de contrôler le démarrage et l'arrêt de la dépense. En sortant de l'orifice, l'eau s'engage dans un canal et se déverse par une trappe coulissante de $96 \mathrm{~cm}$ de long dans le bassin de jauge situé juste en-dessous du canal. L'ouverture et la fermeture de la trappe sont actionnées par un opérateur (voir figure 3). Elles sont presque instantanées ( $1 / 2$ secondes) et les délais se compensent. Une seconde trappe, sous la première, vient compléter le dispositif : ouverte avant la première et fermée après, elle recueille les infiltrations qu'elle déverse dans un petit canal. Enfin, l'eau s'écoulant par la trappe est reçue dans un panier sans fond placé dans le bassin de jauge pour réduire le remous.

Les résultats confirment grosso modo les "lois" déjà connues des expérimentateurs. Ils indiquent que le coefficient de contraction augmente avec la charge sur l'orifice et devient constant, entre 0,6 et 0,625 , quelle que soit, à surface égale, la forme des orifices, pour des charges supérieures à $1,30 \mathrm{~m}$. Les dépenses des mêmes orifices découverts en déversoir sont mesurées pour des charges de fluide comprises entre $2 \mathrm{~cm}$ et $22 \mathrm{~cm}$ de hauteur totale. Poncelet et Lesbros en tirent un coefficient utilisable dans la pratique de 0,7.

Tous ces coefficients de contraction ont été déduits de la mesure des dépenses d'eau. Mais Poncelet et Lesbros s'attaquent aussi à la première méthode, celle de Newton, consistant à calculer directement le coefficient de contraction de la veine liquide. Ils ne peuvent que constater à cet égard l'imperfection des mesures obtenues par leurs prédécesseurs pour l'aire de la veine contractée. Les résultats les plus précis, ceux obtenus par Bidone et Hachette, concernent des ouvertures trop petites, de quelques centimètres carrés. Ils décident donc dès 1827 de reprendre le problème à la base en relevant géométriquement la forme de la veine liquide, et cela pour un orifice carré de $20 \mathrm{~cm}$ de côté et une charge de $1 \mathrm{~m} 68$. Le travail est continué en août et septembre 1828. L'appareil utilisé consiste en un fort châssis de bois auquel est fixé un deuxième châssis intérieur, rectangulaire ou octogone, dont les côtés sont gradués. Une règle de bois, munie d'une tige de fer pointue orientée vers l'intérieur et réglable en longueur, peut coulisser sur chacun de ces côtés. L'appareil est monté parallèlement au plan de l'orifice de telle manière que la veine liquide traverse le châssis intérieur. Pour relever le profil de la veine correspondant en un point, il suffit de régler la tige pour qu'elle effleure la veine en ce point, puis de mesurer la longueur de la tige et la position de la règle sur le châssis (fig. 4).

En procédant ainsi centimètre par centimètre, Lesbros obtient un profil complet. Après avoir relevé neuf profils parallèles au plan de l'orifice, distants les uns des autres de $5 \mathrm{~cm}$, il trace une représentation de la veine liquide en coupe et en plan, à partir de laquelle un modèle en relief est réalisé à l'École d'application par Aimé. De son côté, Poncelet calcule par la méthode de Simpson les aires des différentes sections et trouve un coefficient de contraction inférieur de plus de $6 \%$ à celui obtenue par le calcul de la dépense théorique $\left(0,563 \approx(3 / 4)^{2}\right.$ contre 0,6$)$. Par les mêmes méthodes, Lesbros fait également le profil de la veine sortant d'un déversoir de $20 \mathrm{~cm}$ de largeur et détermine la forme exacte de la dépression au-dessus de l'orifice en fonction de la hauteur des eaux dans le réservoir.

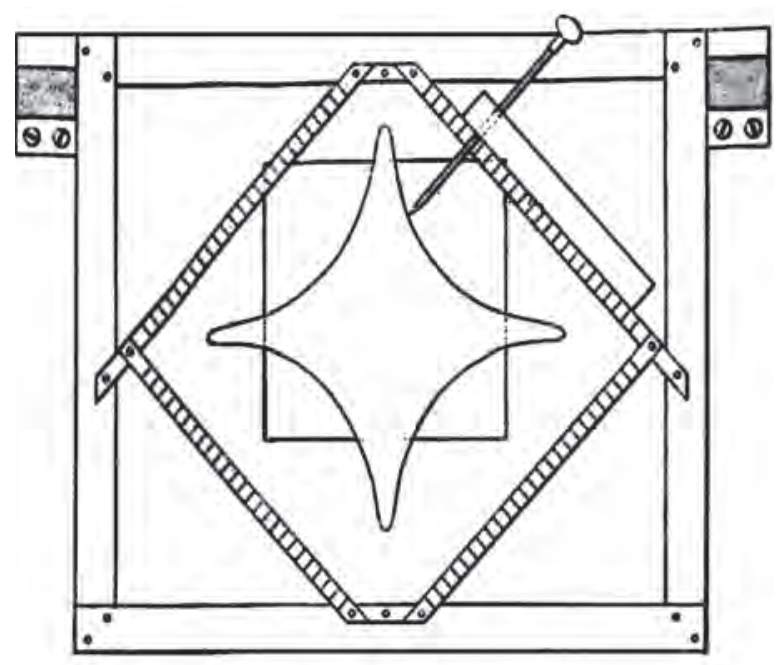

fig. 4 : Appareil pour relever le profil d'une veine 


\section{Postérité}

Les expériences de Poncelet et Lesbros ont été largement diffusées. Elles ont bénéficié de leur caractère officiel et de l'appui du Ministère de la guerre. Poncelet est venu lui-même les présenter à l'Académie des sciences. Son mémoire, rédigé avec le plus grand soin, s'ouvre par un avantpropos expliquant les circonstances qui ont conduit aux expériences. Le premier chapitre expose les conditions générales et les dispositifs d'ensemble. Le chapitre 2 est consacré aux opérations et dispositifs préparatoires. Le chapitre 3, qui donne les résultats, comprend surtout de nombreux tableaux. Enfin, le chapitre 4 contient les réflexions et conclusions de Poncelet. Au total, l'ensemble compte 209 pages, auxquelles s'ajoutent une note de Lesbros, 12 tableaux et 7 planches.

Pourtant, on ne peut pas dire que ce travail ait été un véritable succès. Remarquons d'abord que le mémoire de Poncelet ne concerne que la première partie du programme d'expériences. La deuxième partie, réalisée entre 1829 et 1835, a été laissée à la seule responsabilité de Lesbros. Ses nouvelles expériences concernent cette fois des orifices à parois épaisses prolongées dans certains cas par des coursiers de longueur et d'inclinaison variables et ouverts sur des réservoirs de formes diverses. De tels dispositifs sont beaucoup plus proches des installations d'amenée d'eau que I'on rencontre alors dans l'industrie que ceux des premières expériences. Or, le compte rendu de ces expériences et leurs résultats n'ont été présentés que très tardivement. C'est seulement en 1850 que le ministère de la Guerre transmet à l'Académie des sciences le mémoire préparé et rédigé par Lesbros seul27. Ce long retard s'explique par les multiples occupations professionnelles de l'officier après 1835. Le mémoire de Lesbros remporte le prix de mécanique et il est publié l'année suivante dans la série des Savants étrangers ${ }^{28}$. Mais la

27 Comptes rendus hebdomadaires des séances de l'Académie des sciences, t. 31, $2^{\text {e }}$ semestre 1850, pp. 86-91. Le mémoire, présenté le 22 juillet 1850 à l'Académie, est approuvé le 25 novembre 1850, sur un long rapport rédigé par Poncelet, ibid. pp. 733-742.

28 J. A. Lesbros, «Expériences hydrauliques sur les lois de l'écoulement de l'eau à travers les orifices rectangulaires verticaus de grande dimension ", Mémoires présentés par divers savants à l'Académie des sciences de l'Institut de France, t. 13, 1852, pp. 1-509. Pour le prix de mécanique, Lesbros l'emporte sur Maurel et Jayet qui ont concouru pour un mémoire sur une machine à calculer. Poncelet et Piobert publication intervient trop tard pour avoir exercé beaucoup d'influence sur le développement ultérieur de l'hydraulique pratique.

Du point de vue des progrès de la science, le premier mémoire, celui de Poncelet et Lesbros, est lui aussi décevant. Les coefficients de contraction calculés en jaugeant les dépenses d'eau ne sont que des perfectionnements des résultats déjà obtenus. Quant aux résultats donnés en mesurant directement la contraction de la veine, ils sont plutôt surprenants. Avant Poncelet et Lesbros, les expérimentateurs, comme Michelotti, Venturi, Bossut ou Eytelwein, ont tous obtenu de cette manière un coefficient supérieur à celui résultant de la mesure des quantités dépensées. Certains auteurs ont expliqué cette différence par un ralentissement de vitesse au niveau de l'orifice s'ajoutant à l'effet de la contraction de la veine. La différence en sens contraire, constatée par Lesbros et Poncelet pour des orifices de plus grandes tailles, semble plus difficile à expliquer. Poncelet lui-même, au terme d'une longue analyse, en arrive à rejeter toutes les idées admises sur la vitesse et la pression à l'orifice et au niveau de la veine contractée et à penser que pour déterminer la relation entre vitesse et pression dans la veine, il faudrait, faute de moyens d'expérience, connaître les lois les plus générales du mouvement des fluides ${ }^{29}$ ! Ce constat désabusé, s'il témoigne de sa lucidité, est aussi révélateur des limites du travail effectué.

Car si le mémoire a été admirablement rédigé par Poncelet, s'il rend compte avec un luxe remarquable de détails des expériences menées par Lesbros et s'il fournit des tables très complètes pour les praticiens, il n'apporte aucun résultat notable pour la connaissance des lois de l'écoulement. En particulier, les très longs développements consacrés à l'analyse du phénomène n'aboutissent à aucune conclusion. On n'est donc pas surpris que le mémoire de Poncelet et Lesbros soit rapidement tombé dans l'oubli, y compris chez les historiens de l'hydraulique et de la mécanique des fluides qui ne le citent au mieux qu'en passant. L'ironie est qu'il reste malgré tout quelque chose de ce grand travail pour l'histoire de l'hydrodynamique, mais quelque chose de marginal et qui paraît de détail en comparaison des expériences réalisées. En déterminant le profil de la veine avec leur appareil, Poncelet et Lesbros

sont membres de la commission et Piobert est rapporteur (C.R. Ac. sci., t. 31, pp. 814-815).

29 « Expériences hydrauliques », pp. 396-414. 
ont noté en effet un phénomène inattendu : la formation de rides permanentes, lorsque la pointe de la tige effleure la surface, et qui disparaissent dès que la pointe est légèrement enfoncée. Poncelet rédige une note sur ce phénomène qu'il remet à Arago en décembre 1829. La note, publiée dans les Annales de chimie et de physique en 183130, attire l'attention de scott Russell qui explique le phénomène par la tension de surface du fluide. La théorie de ces rides est finalement donnée en 1871 par Thomson. Ainsi, Poncelet est crédité d'une découverte assez importante, celle des rides capillaires, mais d'une découverte faites un peu par hasard et sans rapport direct avec les expériences qui l'ont permise ${ }^{31}$.

Alors, faut-il reléguer les expériences sur les dépenses d'eau réalisées par Poncelet et Lesbros au vaste cimetière des entreprises scientifiques inutiles? Ce serait oublié leur impact sur la recherche appliquée. Car ce sont ces expériences qui ont ouvert la voie au grand programme de recherche visant à faire revivre à Metz, avec l'appui de la hiérarchie militaire, la tradition de la mécanique expérimentale de Mézières ${ }^{32}$. Si Poncelet en est la principale tête pensante, d'autres officiers militaires, comme Morin et Piobert, y participent activement, avec des expériences sur les effets utiles des moteurs, sur les frottements, sur les tirages des voitures et sur le tir $^{33}$. Plus largement, les expériences de Poncelet et Lesbros témoignent, à leur manière, d'une évolution profonde des conditions de l'expérimentation au début du XIXe siècle. Les historiens de la physique ont souligné l'émergence à partir de la fin du XVIIII siècle d'une nouvelle culture expérimentale, marquée à la fois par un souci de la précision, emprunté à l'astronomie d'observation et à la géodésie, et par

$30 \mathrm{~J}$. V. Poncelet, "Sur quelques phénomènes produits à la surface libre des fluides, en repos ou en mouvement, par la présence des corps solides qui y sont plus ou moins plongés, et spécialement sur les ondulations et les rides permanentes qui en résultent "1, Annales de chimie et de physique, vol. 46, 1831, pp. 5-25.

31 O. Darrigol, Worlds of Flow. A History of Hydrodynamics from the Bernoullis to Prandtl, Oxford, Oxford University Press, p. 88.

32 Pour une mise en contexte de ce programme de recherche, voir P. Bret, L'État, l'armée, la science. L'invention de la recherche publique en France (1763-1830), PUR, Rennes, 2002. 33 Sur les expériences d'A. Morin, voir Cl. Fontanon, "Un ingénieur militaire au service de l'industrialisation : Arthur-Jules Morin (1795-1880)" dans Le Moteur hydraulique en France au XIXe siècle : concepteurs, inventeurs et constructeurs, Cahier d'histoire des sciences et des techniques, $n^{\circ} 29,1990$, pp. 89-117. un souci des applications utiles, empruntés au monde des artisans, des ingénieurs et des administrateurs. Cette culture expérimentale a pris des formes très diverses. Si elle a donné naissance aux sciences de laboratoire, elle s'est exprimée aussi dans les ateliers des fabricants, inventeurs d'instruments de précision, dans les bureaux des administrations, avides de statistiques exactes, et dans les cabinets des ingénieurs, férus de constructions correctement calculées $^{34}$. Dans cette marche vers toujours plus de précision, le travail de Poncelet et Lesbros ouvre un nouveau front : celui des expériences de mécanique en vraie grandeur, qu'exploreront dans les décennies suivantes nombre d'autres ingénieurs et expérimentateurs, en particulier hydrauliciens, à travers l'Europe et jusqu'aux États-Unis, et pour lesquels les Expériences hydrauliques des deux Messins ont pu fournir un modèle ${ }^{35}$.

34 Voir à ce propos, les réflexions d'H. O. Sibum, «Experimentalists in the Republic of Letters ", Science in Context, t. 16, 2003, pp. 89-120.

35 Citons dans la foulée directe de Poncelet et Lesbros, les expériences sur les dépenses d'eau que mène le capitaine Boileau dans son "observatoire hydraulique " de Metz à partir de 1845 et les Lowell hydraulics experiments que J.-B. Francis publie en 1855. 